\title{
CLASSES OF MODULES WITH MANY DIRECT SUMMANDS
}

\author{
I. AL-KHAZZI and P. F. SMITH \\ (Received 20 June 1992; revised 29 September 1992) \\ Communicated by P. Schultz
}

\begin{abstract}
Let $R$ be any ring with identity, $M$ a unital right $R$-module and $\alpha \geq 0$ an ordinal. Then $M$ is a direct sum of a semisimple module and a module having Krull dimension at most $\alpha$ if and only if for every submodule $N$ of $M$ there exists a direct summand $K$ of $M$ such that $K \subseteq N$ and $N / K$ has Krull dimension at most $\alpha$.

1991 Mathematics subject classification (Amer. Math. Soc.): primary 16D70; secondary 16D60, 16P70, $16 \mathrm{P} 20$.
\end{abstract}

It is well known that any finitely generated $\mathbb{Z}$-module is a direct sum of a projective (in fact, a free) module and a module of finite length. More generally, it is proved in [7, Theorem 3.3] that if $R$ is a right Noetherian ring with maximal Artinian right ideal $A$, then every finitely generated right $R$-module is the direct sum of a projective module and a module of finite length if and only if the ideal $A=e R$ for some idempotent $e$ in $R$ and the ring $R / A$ is a (left and right) hereditary (left and right) Noetherian semiprime ring. It was left open in [7] whether the assumption that $R$ be right Noetherian is necessary. In fact, it is not, as Chatters showed, by proving that if $R$ is a ring such that every cyclic right $R$-module is the direct sum of a projective module and a Noetherian module, then $R$ is a right Noetherian ring (see [3, Theorem 3.1])

Chatters [3, Theorem 4.1] also proved that if $\alpha$ is an ordinal and $R$ is a ring such that every cyclic right $R$-module is the direct sum of a projective module and a module of Krull dimension at most $\alpha$, then the right $R$-module $R$ has Krull dimension at most $\alpha+1$. There are other theorems of a similar type. For example, van Huynh and Dan [5] have considered rings with the property that every cyclic right module is the

(C) 1995 Australian Mathematical Society 0263-6115/95 $\$$ A2.00+0.00 
direct sum of a projective module and an Artinian module, or the property that every cyclic right module is the direct sum of a projective module and a semisimple module. This led to the investigations in $[8,9]$.

Let $\mathscr{X}$ be a class of modules. Then $d \mathscr{X}$ is defined in [9] to be the class of modules $M$ such that for each submodule $N$ of $M, N$ is contained in a direct summand $K$ of $M$ such that $K / N$ belongs to $\mathscr{X}$. It is proved in [9, Corollary 3.2] that when $\mathscr{X}$ is the class of Noetherian modules, then a module $M$ belongs to $d \mathscr{X}$ if and only if it can be written as a direct sum of a semisimple module and a Noetherian module. This result generalizes [3, Theorem 3.1].

In this note we will define a class $d^{*} \mathscr{X}$ which is dual to $d \mathscr{X}$, namely a module $M$ belongs to the class $d^{*} \mathscr{X}$ if, for every submodule $N$ of $M$, there exists a direct summand $K$ of $M$ such that $K$ is contained in $N$ and the factor module $N / K$ belongs to $\mathscr{X}$. Our purpose then is to study some properties of $d^{*} \mathscr{X}$ and characterize it in special cases. There are several reasons why this is of interest. In the first place, the results obtained in [8] and [9] are very natural. Secondly, the proofs of these results do not seem to dualize, and therefore proofs for the duals have to be found, if possible. Thirdly, the authors have already had some success in [1], where the dual of a theorem of Goodearl, crucial in the investigations of [8] and [9], is proved.

Here it will be proved that if $R$ is any ring and $\mathscr{X}$ the class of Artinian right $R$-modules, then a module $M$ belongs to the class $d^{*} \mathscr{X}$ if and only if $M$ is the direct sum of a semisimple module and an Artinian module. This is a special case of a result for the class $\mathscr{X}$ of modules with Krull dimension at most $\alpha$, for some ordinal $\alpha \geq 0$ (Theorem 3.5). This fact in turn is proved as a consequence of the fact that if $\mathscr{X}$ is the class of modules such that each homomorphic image has finite uniform dimension, then an $R$-module $M$ belongs to $d^{*} \mathscr{X}$ if and only if $M$ is the direct sum of a semisimple module and a module in the class $\mathscr{X}$ (Theorem 3.4). Moreover, if $R$ is a right FBN-ring and $\mathscr{X}$ the class of right $R$-modules with finite uniform dimension, then a module $M$ belongs to $d^{*} \mathscr{X}$ if and only if $M$ is the direct sum of a semisimple module and a module with finite uniform dimension (Theorem 4.6).

\section{Preliminaries}

Throughout this note, all rings considered have an identity and all modules are unital right modules. For any ring $R$, we define a class $\mathscr{X}$ of $R$-modules to be any collection of $R$-modules such that $\mathscr{X}$ contains a zero module and any module which is isomorphic to some module in $\mathscr{X}$ also belongs to $\mathscr{X}$. Let $M$ be an $R$-module and let $N$ be any submodule of $M$. We call $M$ an $\mathscr{X}$-module if $M$ is a member of the class $\mathscr{X}$. We call $N$ an $\mathscr{X}$-submodule of $M$ if $N$ is an $\mathscr{X}$-module. Following the terminology in [9], a class $\mathscr{X}$ is called $s$-closed if every submodule of an $\mathscr{X}$-module 
is an $\mathscr{X}$-module.

Let $\mathscr{X}$ and $\mathscr{Y}$ be classes of modules over the same ring $R$. Then $\mathscr{X} \oplus \mathscr{Y}$ is defined to be the class of modules $M$ such that $M$ is the direct sum of an $\mathscr{X}$-submodule and a $\mathscr{Y}$-submodule. It is clear that any $\mathscr{X}$-module is an $(\mathscr{X} \oplus \mathscr{Y})$-module, and $\mathscr{X} \oplus \mathscr{Y}=\mathscr{Y} \oplus \mathscr{X}$.

Let $\mathscr{X}$ be any class of modules. The class $d \mathscr{X}$ consists of all modules $M$ such that, for every submodule $N$ of $M$ there exists a direct summand $K$ of $M$ such that $N \subseteq K$ and the factor module $K / N$ is an $\mathscr{X}$-module. Properties of the class $d \mathscr{X}$ are given in [8] and [9].

We define a class $d^{*} \mathscr{X}$ dual to $d \mathscr{X}$. Indeed, $d^{*} \mathscr{X}$ is defined to be the class of $R$-modules $M$ such that each submodule $N$ of $M$ contains a direct summand $K$ of $M$ such that the factor module $N / K$ is an $\mathscr{X}$-module.

For any ring $R$, we denote the classes of zero modules, simple modules, semisimple modules, injective modules and modules of finite uniform dimension by

$$
\mathscr{Z}, \mathscr{S}, \mathscr{C}, \mathscr{I}, \mathscr{U}
$$

respectively. It will always be clear from the context which ring $R$ is being considered. Any unexplained terminology can be found in [2].

\section{General properties}

Throughout this section, we shall consider classes of modules over any ring $R$. We establish some facts which will be useful in the proofs of the theorems in the next section and also give some general properties of the class $d^{*} \mathscr{X}$, where $\mathscr{X}$ is a given class of right $R$-modules. The following elementary result gives an alternative characterization of the class $d^{*} \mathscr{X}$.

LEMMA 2.1. Let $\mathscr{X}$ be any class of modules. Then a module $M$ belongs to $d^{*} \mathscr{X}$ if and only if, for every submodule $N$ of $M$, there exist a direct summand $K$ of $M$ and an $\mathscr{X}$-submodule $L$ of $M$ such that $N=K \oplus L$.

PROOF. The sufficiency is clear. Conversely, suppose that $M \in d^{*} \mathscr{X}$ and $N$ is a submodule of $M$. By hypothesis, there exist submodules $K$ and $K^{\prime}$ of $M$ such that $M=K \oplus K^{\prime}, K \subseteq N$ and $N / K \in \mathscr{X}$. By the Modular Law, $N=K \oplus\left(N \cap K^{\prime}\right)$. Note that $N \cap K^{\prime} \cong N / K \in \mathscr{X}$. Thus $M$ has the stated condition.

LEMMA 2.2. Let $\mathscr{X}$ and $\mathscr{Y}$ be classes of modules such that $\mathscr{X} \subseteq \mathscr{Y}$. Then

(i) $d^{*} \mathscr{X} \subseteq d^{*} \mathscr{Y}$,

(ii) $\mathscr{C}=d^{*} \mathscr{Z}=d^{*} \mathscr{I} \subseteq d^{*} \mathscr{X}=d^{*}(\mathscr{I} \oplus \mathscr{X})$, and 
(iii) $d^{*} \mathscr{X}$ is $s$-closed.

PROOF. Claim (i) is clear.

To prove (ii), note that by [2, Theorem 9.6], $\mathscr{C}=d^{*} \mathscr{Z} \subseteq d^{*} \mathscr{I}$. Let $M$ be a $d^{*} \mathscr{I}$-module and let $N$ be a submodule of $M$. There exist submodules $K$ and $K^{\prime}$ of $M$ such that $M=K \oplus K^{\prime}, K \subseteq N$ and $N / K$ is injective. Thus $N \cap K^{\prime}$ is injective and hence $N \cap K^{\prime}$ is a direct summand of $K^{\prime}$. Thus $N$ is a direct summand of $M$. By [2, Theorem 9.6], $M$ is a $\mathscr{C}$-module.

Now (i) gives $d^{*} \mathscr{Z} \subseteq d^{*} \mathscr{X} \subseteq d^{*}(\mathscr{I} \oplus \mathscr{X})$. Let $M \in d^{*}(\mathscr{I} \oplus \mathscr{X})$. Let $N$ be any submodule of $M$. Then there exist submodules $K$ and $K^{\prime}$ of $M$ such that $M=K \oplus K^{\prime}, K \subseteq N$ and $N / K$ is an $(\mathscr{I} \oplus \mathscr{X})$-module. Now $N=K \oplus\left(N \cap K^{\prime}\right)$ and $N \cap K^{\prime}$ has the form $I \oplus L$ for some injective submodule $I$ and $\mathscr{X}$-submodule $L$, because $N \cap K^{\prime} \cong N / K$. Then $I$ is a direct summand of $K^{\prime}$ and hence $K \oplus I$ is a direct summand of $M$. Moreover, $N /(K \oplus I) \cong L \in \mathscr{X}$. It follows that $M \in d^{*} \mathscr{X}$. Therefore $d^{*}(\mathscr{I} \oplus \mathscr{X}) \subseteq d^{*} \mathscr{X}$.

To prove (iii), let $M$ be a $d^{*} \mathscr{X}$-module. Let $N$ be a submodule of $M$ and $H$ be a submodule of $N$. By Lemma $2.1, H=K \oplus L$ for some direct summand $K$ of $M$ and $\mathscr{X}$-submodule $L$ of $M$. But $K$ is a direct summand of $N$. Thus $N$ is a $d^{*} \mathscr{X}$-module, by Lemma 2.1 . It follows that $d^{*} \mathscr{X}$ is $s$-closed.

PROPOSITION 2.3. Let $\mathscr{X}$ be any class of modules. Then

(i) $\mathscr{C} \oplus d \mathscr{X}=d \mathscr{X}$

(ii) $\mathscr{C} \oplus d^{*} \mathscr{X}=d^{*} \mathscr{X}$.

PROOF. (i) Clearly, $d \mathscr{X} \subseteq \mathscr{C} \oplus d \mathscr{X}$. Now suppose that $M \in \mathscr{C} \oplus d \mathscr{X}$. Then $M=M_{1} \oplus M_{2}$, where $M_{1}$ is a $\mathscr{C}$-module and $M_{2}$ a $\mathscr{X}$-module. Let $N$ be a submodule of $M$. Then

$$
N+M_{2}=\left[\left(N+M_{2}\right) \cap M_{1}\right] \oplus M_{2} .
$$

But $\left(N+M_{2}\right) \cap M_{1}$ is a direct summand of $M_{1}$. Therefore $N+M_{2}$ is a direct summand of $M$.

Since $N \cap M_{2}$ is a submodule of $M_{2}$, it follows that $M_{2}=K \oplus K^{\prime}$ for some submodules $K$ and $K^{\prime}$ such that $N \cap M_{2} \subseteq K$ and $K /\left(N \cap M_{2}\right)$ is an $\mathscr{X}$-module. Note that

$$
\begin{aligned}
(K+N) \cap K^{\prime} & =\left[(K+N) \cap M_{2}\right] \cap K^{\prime} \\
& =\left[K+\left(N \cap M_{2}\right)\right] \cap K^{\prime}=K \cap K^{\prime}=0 .
\end{aligned}
$$

Clearly, $(K+N)+K^{\prime}=N+M_{2}$. Thus

$$
N+M_{2}=(K+N) \oplus K^{\prime},
$$


and hence $K+N$ is a direct summand of $M$. Now $N \subseteq K+N$, and $(K+N) / N \cong$ $K /\left(N \cap M_{2}\right)$. Hence $(K+N) / N$ is an $\mathscr{X}$-module. It follows that $M$ is a $d \mathscr{X}$-module.

(ii) It is clear that $d^{*} \mathscr{X} \subseteq \mathscr{C} \oplus d^{*} \mathscr{X}$. Now consider any $\left(\mathscr{C} \oplus d^{*} \mathscr{X}\right)$-module $M$. The module $M=M_{1} \oplus M_{2}$ for some $\mathscr{C}$-module $M_{1}$ and $d^{*} \mathscr{X}$-module $M_{2}$. Let $N$ be a submodule of $M$. Then $M_{1}=\left(N \cap M_{1}\right) \oplus M^{\prime}$ for some submodule $M^{\prime}$ of $M_{1}$. Thus $M=\left(N \cap M_{1}\right) \oplus M^{\prime} \oplus M_{2}$, and $N=\left(N \cap M_{1}\right) \oplus H$, where $H=N \cap\left(M^{\prime} \oplus M_{2}\right)$. Since $\left(M_{2} \oplus M^{\prime}\right) / M^{\prime}$ is a $d^{*} \mathscr{X}$-module, it follows that $\left(H+M^{\prime}\right) / M^{\prime}=\left(K / M^{\prime}\right) \oplus\left(L / M^{\prime}\right)$ for some submodules $K$ and $L$ containing $M^{\prime}$, such that $K / M^{\prime}$ is a direct summand of $\left(M_{2} \oplus M^{\prime}\right) / M^{\prime}$ and $L / M^{\prime}$ is an $\mathscr{X}$-module, by Lemma 2.1. Thus $K$ is a direct summand of $M$. But $K=M^{\prime} \oplus(K \cap H)$, so that $K \cap H$ is also a direct summand of $M$. It is now clear that $\left(N \cap M_{1}\right) \oplus(K \cap H)$ is a direct summand of $M$. Moreover,

$$
\begin{aligned}
& N /\left[\left(N \cap M_{1}\right) \oplus(K \cap H)\right] \cong H /(K \cap H) \cong(H+K) / K \\
& \quad=\left(H+M^{\prime}\right) / K \cong L / M^{\prime} \in \mathscr{X} .
\end{aligned}
$$

It follows that $M \in d^{*} \mathscr{X}$. Thus $\mathscr{C} \oplus d^{*} \mathscr{X} \subseteq d^{*} \mathscr{X}$.

COROLlaRY 2.4. Let $\mathscr{X}$ be any s-closed class of modules. Then $\mathscr{C} \oplus \mathscr{X} \subseteq d^{*} \mathscr{X}$.

Proof. Because $\mathscr{X}$ is $s$-closed, $\mathscr{X} \subseteq d^{*} \mathscr{X}$ and hence, by Proposition 2.3(ii), $\mathscr{C} \oplus \mathscr{X} \subseteq \mathscr{C} \oplus d^{*} \mathscr{X}=d^{*} \mathscr{X}$.

Let $R$ be a ring. Let $\mathscr{X}$ be a class of $R$-modules. For any $R$-module $M$, we define the $\mathscr{X}$-socle Soc $\mathscr{X}(M)$ to be the sum of all $\mathscr{X}$-submodules of $M$. Clearly $M=\operatorname{Soc}_{\mathscr{X}}(M)$ if $M \in \mathscr{X}$. If $\mathscr{X}=\mathscr{S}$ then $\operatorname{Soc}_{\mathscr{S}}(M)$ is the usual socle of $M$ and is denoted simply by Soc $M$. Now we investigate the internal structure of $d^{*} \mathscr{X}$-modules.

PROPOSITION 2.5. Let $\mathscr{X}$ be any class of modules. Let $M$ be any $d^{*} \mathscr{X}$-module. Then the module $M / \operatorname{Soc} \mathscr{X}(M)$ is semisimple.

PROOF. Let $S=\operatorname{Soc} \mathscr{X}(M)$. Any submodule of $M / S$ has the form $N / S$ for some submodule $N$ of $M$ containing $S$. There exists submodules $K$ and $K^{\prime}$ of $M$ such that $M=K \oplus K^{\prime}, K \subseteq N$ and $N / K \in \mathscr{X}$. Hence $N=K \oplus\left(N \cap K^{\prime}\right)$ and $N \cap K^{\prime} \in \mathscr{X}$. Thus $N \cap K^{\prime} \subseteq S$, and we deduce that $M / S=(N / S) \oplus\left[\left(K^{\prime}+S\right) / S\right]$. That is, $N / S$ is a direct summand of $M / S$. By [2, Theorem 9.6], $M / S$ is semisimple.

COROLlaRY 2.6. Let $\mathscr{X}$ be any class of modules such that $\mathscr{S} \subseteq \mathscr{X}$. Let $M$ be any $d^{*} \mathscr{X}$-module. Then $\operatorname{Soc} \mathscr{X}(M)$ is an essential submodule of $M$. 
ProOF. Let $N$ be any submodule of $M$ such that $N \cap \operatorname{Soc} \mathscr{X}(M)=0$. Then $N$ embeds in $M / \operatorname{Soc}_{\mathscr{X}}(M)$. By Proposition $2.5, N \in \mathscr{C}$, so that, by hypothesis,

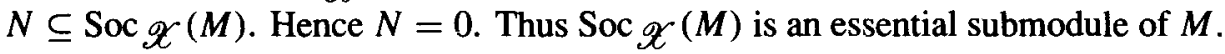

LEMMA 2.7. Let $\mathscr{X}$ be any class of modules. Let $M$ be a $d^{*} \mathscr{X}$-module and let $N$ be any submodule of $M$. Then $N$ contains a non-zero $\mathscr{X}$-submodule or $N$ is a semisimple direct summand of $M$.

ProOF. Suppose that $N$ does not contain a non-zero $\mathscr{X}$-submodule. Let $P$ be any submodule of $N$. By Lemma 2.1, $P=K \oplus L$ for some direct summand $K$ of $M$ and $\mathscr{X}$-submodule $L$ of $M$. But $L=0$, and hence, $P=K$. By [2, Theorem 9.6], $N$ is a semisimple direct summand of $M$.

PROPOSITION 2.8. Let $\mathscr{X}$ be any s-closed class of modules and let $M$ be any $d^{*} \mathscr{X}$ module. Then there exist a semisimple submodule $M_{1}$ and a submodule $M_{2}$ of $M$ such that $M=M_{1} \oplus M_{2}$ and every non-zero submodule of $M_{2}$ contains a non-zero $\mathscr{X}$-submodule.

PROOF. By Zorn's Lemma, $M$ contains a submodule $M_{1}$ maximal with respect to the property that it does not contain a non-zero $\mathscr{X}$-submodule. By Lemma 2.7, $M_{1}$ is a semisimple direct summand of $M$. There exists a submodule $M_{2}$ such that $M=M_{1} \oplus M_{2}$. Let $N$ be a non-zero submodule of $M_{2}$. Then $M_{1} \oplus N$ contains a non-zero $\mathscr{X}$-submodule $K$, by the choice of $M_{1}$. Note that $K \cap M_{1} \in \mathscr{X}$ and hence $K \cap M_{1}=0$. Thus $K$ embeds in $N$ and hence $N$ contains a non-zero $\mathscr{X}$-submodule.

\section{The main theorem}

Let $R$ be any ring. Let $M$ be a right $R$-module. By a subquotient of $M$ we shall mean a right $R$-module $N / K$, where $K \subseteq N$ are submodules of $M$. Recall that $\mathscr{U}$ denotes the class of right $R$-modules with finite uniform dimension. Adopting the notation of [8] and [9], we denote by $h \mathscr{U}$ the class of modules $M$ such that every homomorphic image is a $\mathscr{U}$-module. We next give an easy characterization of $(h \mathscr{U})$-modules.

LEMMA 3.1. Let $R$ be any ring. Then an $R$-module $M$ is an ( $h \mathscr{U})$-module if and only if every semisimple subquotient of $M$ is finitely generated.

PROOF. The necessity is clear. Conversely, suppose that $M$ is not an $(h \mathscr{U})$-module. Then there exists a submodule $K$ of $M$ such that $M / K \notin \mathscr{U}$. There exist elements 
$x_{n} \in M \backslash K(n \geq 1)$ such that the sum $\left[\left(x_{1} R+K\right) / K\right]+\left[\left(x_{2} R+K\right) / K\right]+\cdots$ is direct. For each $n \geq 1, K$ is contained in a maximal submodule $P_{n}$ of $\left(x_{n} R+K\right)$. Let $N=K+x_{1} R+x_{2} R+\cdots$ and let $P=P_{1}+P_{2}+P_{3}+\cdots$. Then $P$ is a submodule of $N$ and $N / P \cong \bigoplus_{n \geq 1}\left(x_{n} R+K\right) / P_{n}$. Therefore $N / P$ is a non-finitely generated semisimple subquotient of $M$.

COROLlaRY 3.2. Let $R$ be a ring and $M$ an $R$-module. Let $N$ be a submodule of $M$. Then $M$ is an ( $h \mathscr{U})$-module if and only if $N$ and $M / N$ are both $(h \mathscr{U})$-modules.

ProOF. The necessity is clear (for example, by Lemma 3.1). Conversely suppose that $N$ and $M / N$ are both $(h \mathscr{U})$-modules. Let $P \subseteq Q$ be submodules of $M$ such that $Q / P$ is semisimple. We shall prove that $Q / P$ is finitely generated. Note that

$$
Q /[P+(Q \cap N)] \cong(Q+N) /(P+N)
$$

so that $Q /[P+(Q \cap N)]$ is finitely generated. Moreover,

$$
[P+(Q \cap N)] / P \cong(Q \cap N) /(P \cap N),
$$

so that $[P+(Q \cap N)] / P$ is finitely generated. It follows that $Q / P$ is finitely generated. By Lemma 3.1, $M \in h \mathscr{U}$.

Let $R$ be any ring and let $\mathscr{X}$ be any class of modules over $R$. Then $a \mathscr{X}$ will denote the class of modules such that every proper submodule is an $\mathscr{X}$-module. It is clear that $a \mathscr{X} \subseteq d^{*} \mathscr{X}$, for any class $\mathscr{X}$. We also define, for any positive integer $n$, $\mathscr{X}^{(n)}=\mathscr{X} \oplus \mathscr{X} \oplus \cdots \oplus \mathscr{X}$ ( $n$ summands). We define

$$
\mathscr{X}^{(\omega)}=\bigcup_{n \geq 1} \mathscr{X}^{(n)}
$$

LEMMA 3.3. Let $\mathscr{X}$ be any class of modules and let $\mathscr{Y}=h \mathscr{U}$. Then

(i) $\left(\mathscr{U} \cap d^{*} \mathscr{X}\right) \subseteq(a \mathscr{X})^{(\omega)}$.

(ii) $\mathscr{Y}=(a \mathscr{Y})^{(\omega)}$.

(iii) $\mathscr{U} \cap d^{*} \mathscr{Y}=\mathscr{Y}$.

PROOF. (i) Let $M \in \mathscr{U} \cap d^{*} \mathscr{X}$. Then $M=M_{1} \oplus \cdots \oplus M_{n}$ is a finite direct sum of indecomposable submodules $M_{i}(1 \leq i \leq n)$. By Lemma 2.2(iii), $M_{i} \in d^{*} \mathscr{X}$ and hence $M_{i} \in a \mathscr{X}$ for each $1 \leq i \leq n$. Thus $M \in(a \mathscr{X})^{(\omega)}$.

(ii) Clearly $\mathscr{Y}=a \mathscr{Y}$, by Lemma 3.1. Moreover, $\mathscr{Y}=\mathscr{Y}^{(\omega)}$. This proves (ii).

(iii) This follows directly from parts (i) and (ii). 
It is proved in $[8$, Theorem 3.1$]$ that

$$
d \mathscr{U}=d(h \mathscr{U})=\mathscr{C} \oplus(h \mathscr{U}) .
$$

Now we prove the following analogue of this fact.

THEOREM 3.4. For any ring $R, d^{*}(h \mathscr{U})=\mathscr{C} \oplus(h \mathscr{U})$.

PROOF. By Corollary $2.4, \mathscr{C} \oplus(h \mathscr{U}) \subseteq d^{*}(h \mathscr{U})$. Conversely, let $M \in d^{*}(h \mathscr{U})$. Then there exist submodules $K, K^{\prime}$ of $M$ such that $M=K \oplus K^{\prime}, K \subseteq$ Soc $M$ and $(\operatorname{Soc} M) / K \in h \mathscr{U}$. Note that $K \in \mathscr{C}$. Moreover, Soc $K^{\prime} \cong(\operatorname{Soc} M) / K$, so that Soc $K^{\prime}$ is finitely generated. By Lemma 2.2(iii), we can suppose, without loss of generality, that Soc $M$ is finitely generated, and prove that $M \in h \mathscr{U}$.

Let $P \subseteq Q$ by submodules of $M$ such that $Q / P \in \mathscr{C}$. Lemma 2.2(iii) allows us to assume $M=Q$. There exist submodules $L, L^{\prime}$ of $M$ such that $M=L \oplus L^{\prime}, L \subseteq P$ and $P / L \in h \mathscr{U}$. Note that $P=L \oplus\left(P \cap L^{\prime}\right)$, and hence $\left(P \cap L^{\prime}\right) \cong P / L \in \mathscr{U}$. By Zorn's Lemma, there exists a submodule $L^{\prime \prime}$ of $L^{\prime}$ maximal with respect to the property $L^{\prime \prime} \cap\left(P \cap L^{\prime}\right)=0$. Note that $L^{\prime \prime} \cap P=0$ gives $L^{\prime \prime}$ is semisimple, and hence finitely generated, because Soc $M$ is finitely generated. The submodule $L^{\prime}$ contains the essential submodule $L^{\prime \prime} \oplus\left(P \cap L^{\prime}\right)$ which has finite uniform dimension. Therefore $L^{\prime} \in \mathscr{U}$. Now Lemmas 2.2(iii) and 3.3(iii) together give $L^{\prime} \in \mathscr{U} \cap d^{*}(h \mathscr{U})=h \mathscr{U}$. However, $M / L \cong L^{\prime}$, and hence $M / P$ is finitely generated. Therefore by Lemma 3.1, $M \in h \mathscr{U}$.

Let $R$ be any ring. For any ordinal $\alpha \geq 0$, let $\mathscr{K}_{\alpha}$ denote the class of modules with Krull dimension at most $\alpha$. Note that $\mathscr{K}_{0}$ is the class of Artinian modules. In [9, Corollary 3.2] it is proved that $d \mathscr{N}=\mathscr{C} \oplus \mathscr{N}$, for any ring $R$, where $\mathscr{N}$ denotes the class of Noetherian modules. We now prove the dual of this result.

THEOREM 3.5. For any ring $R$ and ordinal $\alpha \geq 0, d^{*} \mathscr{K}_{\alpha}=\mathscr{C} \oplus \mathscr{K}_{\alpha}$.

Proof. By Corollary 2.4, $\mathscr{C} \oplus \mathscr{K}_{\alpha} \subseteq d^{*} \mathscr{K}_{\alpha}$. Conversely, suppose that $M \in$ $d^{*} \mathscr{K}_{\alpha}$. Because $\mathscr{K}_{\alpha} \subseteq h \mathscr{U}$ (see, for example, [4, Lemma 1.1 and Proposition 1.4]), Lemma 2.2(i) and Theorem 3.4 give that $M=M_{1} \oplus M_{2}$ for some $\mathscr{C}$-module $M_{1}$, and $\mathscr{U}$-module $M_{2}$. By Lemma 2.2(iii), $M_{2} \in d^{*} \mathscr{K}_{\alpha}$, and, by Lemma 3.3(i), $M_{2} \in$ $\left(a \mathscr{K}_{\alpha}\right)^{(\omega)}$. But, clearly $a \mathscr{K}_{\alpha}=\mathscr{K}_{\alpha}$ (see [4, Lemma 1.1]). Thus $M_{2} \in \mathscr{K}_{\alpha}$. It follows that $M \in \mathscr{C} \oplus \mathscr{K}_{\alpha}$.

In particular, Theorem 3.5 asserts that $d^{*} \mathscr{A}=\mathscr{C} \oplus \mathscr{A}$, for any ring $R$, where $\mathscr{A}$ is the class of Artinian $R$-modules. 


\section{FBN-rings}

Let $R$ be a ring. We know from [8, Theorem 3.1] that

$$
d \mathscr{U}=d(h \mathscr{U})=\mathscr{C} \oplus(h \mathscr{U}) .
$$

Clearly $d^{*}(h \mathscr{U}) \subseteq d^{*} \mathscr{U}$. However, if $R=K\left[X_{1}, X_{2}, \ldots\right]$ denotes the polynomial ring over a field $K$ in a countably infinite number of commuting indeterminates $X_{1}, X_{2}, \ldots$, then $R_{R}$ is uniform, so that $R_{R} \in d^{*} \mathscr{U}$, but $R_{R} \notin \mathscr{C} \oplus h \mathscr{U}=d^{*}(h \mathscr{U})$, because $R_{R}$ has zero socle and the ring $R$ has a homomorphic image with non-finitely generated socle. Thus $d^{*}(h \mathscr{U}) \neq d^{*} \mathscr{U}$.

This raises the question: given a ring $R$, what is $d^{*} \mathscr{U}$ for $R$ ? We shall show that $d^{*} \mathscr{U}=\mathscr{C} \oplus \mathscr{U}$ for the class of right FBN-rings.

Let $R$ be any ring. Let $\mathscr{X}$ and $\mathscr{Y}$ be classes of $R$-modules. Then $\mathscr{X} \mathscr{Y}$ is defined to be the class of modules $M$ which contains an $\mathscr{X}$-submodule $N$ such that $M / N$ is a $\mathscr{Y}$-module. For any ring $R$, we denote the class of finitely generated modules by $\mathscr{G}$.

PROPOSITION 4.1. For any ring $R, d^{*} \mathscr{U} \cap \mathscr{C} \mathscr{G} \subseteq \mathscr{C} \oplus \mathscr{U}$.

ProOF. Let $M \in d^{*} \mathscr{U} \cap \mathscr{C} \mathscr{G}$. Let $S$ denote the socle Soc $M$ of $M$. There exists a $\mathscr{C}$-submodule $N$ of $M$ such that $M / N$ is finitely generated. Therefore $M / S$ is finitely generated. Hence there exists a finitely generated submodule $L$ of $M$ such that $M=L+S$. Since $S$ is semisimple, we know that $S=(L \cap S) \oplus S_{1}$ for some submodule $S_{1}$ of $S$. Therefore, $S_{1}$ is semisimple and $M=L \oplus S_{1}$.

Suppose that $L$ is not a $\mathscr{U}$-module. Then there exists a submodule $N$ of $L$ such that $N$ is an infinite direct sum of non-zero submodules of $L$. Now by Lemma 2.2(iii), $L \in d^{*} \mathscr{U}$, and, by Lemma 2.1, there exist a direct summand $K$ of $L$ and a $\mathscr{U}$ submodule $P$ such that $N=K \oplus P$. But $L$ finitely generated implies $K$ is finitely generated. This fact combined with the fact that $P$ has finite uniform dimension shows that $N$ is not an infinite direct sum of non-zero submodules, a contradiction. Therefore $L$ is a $\mathscr{U}$-module. Thus $M \in \mathscr{C} \oplus \mathscr{U}$.

COROLLARY 4.2. Let $R$ be any ring and $M$ any $d^{*} \mathscr{U}$-module. Then any finitely generated submodule of $M$ has finite uniform dimension.

PROOF. By Lemma 2.2(iii) and Proposition 4.1.

LEMMA 4.3. Let $R$ be any ring. Let $M$ be a $d^{*} \mathscr{U}$-module with zero socle. Then $M$ has finite uniform dimension or there exist a countable family of independent cyclic uniform submodules $N_{i}(i \geq 1)$, proper non-zero submodules $K_{i}$ of $N_{i}(i \geq 1)$ and independent submodules $L, L^{\prime}$ of $M$ such that 
(a) $L \oplus L^{\prime}=N_{1} \oplus N_{2} \oplus N_{3} \oplus \cdots$,

(b) $L \subseteq K_{1} \oplus K_{2} \oplus K_{3} \oplus \cdots$, and

(c) L' contains a finitely generated essential submodule.

ProOF. Suppose $M \notin \mathscr{U}$. Let $N=N_{1} \oplus N_{2} \oplus N_{3} \oplus \cdots$ be an infinite direct sum of non-zero cyclic submodules of $M$. By Corollary 4.2 we can suppose without loss of generality that $N_{i}$ is uniform for each $i \geq 1$. For each $i \geq 1, N_{i}$ has zero socle and hence $N_{i}$ contains a proper non-zero (that is, essential) submodule $K_{i}$ (see [2, Proposition 9.7]). Let $K$ denote the submodule $K_{1} \oplus K_{2} \oplus K_{3} \oplus \cdots$ of $N$ and note that $K$ is essential in $N$ (see [2, Proposition 5.20]). By hypothesis and Lemma 2.2(iii), there exist submodules $L$ and $L^{\prime}$ of $N$ such that $N=L \oplus L^{\prime}$ with $L \subseteq K$ and $K / L$ a $\mathscr{U}$-module. Note that $K=L \oplus\left(K \cap L^{\prime}\right), K \cap L^{\prime} \cong K / L$ and hence $K \cap L^{\prime}$ is a $\mathscr{U}$-module. But $K$ essential in $N$ implies $K \cap L^{\prime}$ is essential in $L^{\prime}$. This implies $L^{\prime}$ is a $\mathscr{U}$-module. Therefore $L^{\prime}$ contains a finitely generated essential submodule.

COROLlARY 4.4. Let $R$ be any ring. Then any non-singular $d^{*} \mathscr{U}$-module with zero socle has finite uniform dimension.

PROOF. Suppose that $M$ is a non-singular $d^{*} \mathscr{U}$-module with zero socle and $M \notin \mathscr{U}$. By Lemma 4.3 there exist submodules $N_{i}(i \geq 1), K_{i}(i \geq 1), L$ and $L^{\prime}$ of $M$ with the properties listed in the lemma. Let $H$ be a finitely generated essential submodule of $L^{\prime}$. Because $H$ is finitely generated, there exists $t \geq 1$ such that $H \subseteq N_{1} \oplus \cdots \oplus N_{t}$.

Let $x \in N_{t+1}, x \notin K_{t+1}$. Then $x=y-y^{\prime}$ for some $y \in L, y^{\prime} \in L^{\prime}$. Note that $y \in K$ so that $y=k_{1}+\cdots+k_{n}$ for some $n \geq 1, k_{i} \in K(1 \leq i \leq n)$. There exists an essential right ideal $E$ of $R$ such that $y^{\prime} E \subseteq H \subseteq N_{1} \oplus \cdots \oplus N_{t}$. Now $y^{\prime}=y-x=k_{1}+\cdots+k_{t}+\left(k_{t+1}-x\right)+k_{t+2}+\cdots+k_{n}$. However, $y^{\prime} E \subseteq N_{1} \oplus \cdots \oplus N_{t}$ implies that $\left(k_{t+1}-x\right) E=0$. Recall that $N_{t+1}$ is non-singular. Thus $k_{t+1}-x=0$. Hence $x=k_{t+1} \in K_{t+1}$, a contradiction. It follows that $M$ is a $\mathscr{U}$-module.

For any ring $R$, let $\mathscr{T}$ denote the class of singular modules.

PROPOSITION 4.5. For any ring $R, \mathscr{C} \oplus \mathscr{U} \subseteq d^{*} \mathscr{U} \subseteq \mathscr{T} \oplus \mathscr{C} \oplus \mathscr{U}$.

PROOF. By Corollary $2.4, \mathscr{C} \oplus \mathscr{U} \subseteq d^{*} \mathscr{U}$. Now suppose that $M$ is a $d^{*} \mathscr{U}$-module. Let $S$ be the socle of $M$. Then there exist submodules $K$ and $K^{\prime}$ of $M$ such that $M=K \oplus K^{\prime}, K \subseteq S$ and Soc $K^{\prime}=S \cap K^{\prime}$ is a $\mathscr{U}$-module. It follows that $K$ is a $\mathscr{C}$-module and $K^{\prime}$ has finitely generated socle $S \cap K^{\prime}$. Let $Z=Z\left(K^{\prime}\right)$, the singular submodule of $K^{\prime}$. By Lemma 2.2(iii), $K^{\prime}$ is a $d^{*} \mathscr{U}$-module and hence there exist submodules $L$ and $L^{\prime}$ of $K^{\prime}$ such that $K^{\prime}=L \oplus L^{\prime}, L \subseteq Z$ and $Z \cap L^{\prime}$ is a $\mathscr{U}$-module. Thus $L$ is singular and the singular submodule $Z^{\prime}$ of $L^{\prime}$ is a $\mathscr{U}$-module. 
We claim that $L^{\prime}$ is a $\mathscr{U}$-module. Suppose not. Then there exists an infinite direct sum $P=P_{1} \oplus P_{2} \oplus P_{3} \oplus \cdots$ of non-zero submodules of $L^{\prime}$.

Because the socle of $\mathrm{K}^{\prime}$ is a $\mathscr{U}$-module, and $Z^{\prime}$ is a $\mathscr{U}$-module we can suppose without loss of generality that $P_{i}$ is non-singular with zero socle for all $i \geq 1$. Thus $P$ is a non-singular $d^{*} \mathscr{U}$-module with zero socle. By Corollary 4.4,P is a $\mathscr{U}$-module, a contradiction. Thus $L^{\prime}$ is a $\mathscr{U}$-module. It follows that

$$
M=L \oplus K \oplus L^{\prime} \in \mathscr{T} \oplus \mathscr{C} \oplus \mathscr{U} .
$$

Using Proposition 4.5 we shall prove that for any commutative Noetherian ring $R, d^{*} \mathscr{U}=\mathscr{C} \oplus \mathscr{U}$. In fact we can do rather better. Recall that a ring $R$ is a right FBN-ring provided $R$ is right Noetherian and, for every prime homomorphic image $S$ of $R$, every essential right ideal of $S$ contains a non-zero two-sided ideal. Examples of right FBN-rings are commutative Noetherian rings and right Noetherian rings which satisfy a polynomial identity (that is, right Noetherian PI-rings).

Let $R$ be a right Noetherian ring and $U$ a uniform right $R$-module. Recall that

$$
P=\{r \in R: A r=0 \text { for some non-zero submodule } A \text { of } U\}
$$

is a prime ideal of $R$ and $P=\operatorname{ann}(W)$, the annihilator of some non-zero submodule $W$ of $U$. We call $P$ the assassinator of $U$ and write $P=\operatorname{ass}(U)$.

THEOREM 4.6. Let $R$ be a right FBN-ring. Then $d^{*} \mathscr{U}=\mathscr{C} \oplus \mathscr{U}$.

Proof. By Corollary 2.4, $\mathscr{C} \oplus \mathscr{U} \subseteq d^{*} \mathscr{U}$. Suppose that $d^{*} \mathscr{U} \neq \mathscr{C} \oplus \mathscr{U}$ for the ring $R$. Because $R$ is right Noetherian, we can suppose that $d^{*} \mathscr{U}=\mathscr{C} \oplus \mathscr{U}$ for any proper homomorphic image of $R$.

Let $M$ be a $d^{*} \mathscr{U}$-module such that $M$ is not a $(\mathscr{C} \oplus \mathscr{U})$-module. By Proposition 4.5, $M \in \mathscr{T} \oplus \mathscr{C} \oplus \mathscr{U}$. By Lemma 2.2(iii), we can suppose without loss of generality that $M$ is singular. By Lemma 4.3, $M$ contains submodules $N_{i}(i \geq 1), K_{i}(i \geq 1)$, $L$ and $L^{\prime}$ with the properties stated in the lemma. For each $i \geq 1$, let $P_{i}=\operatorname{ass}\left(N_{i}\right)$. Clearly we can suppose without loss of generality that $N_{i} P_{i}=0$. Moreover, because $N_{i}$ is singular and $R$ is a right FBN-ring, $P_{i}$ is an essential right ideal of $R$ for each $i \geq 1$ (see[6, 6.10.4]).

There exists a finitely generated essential submodule $H$ of $L^{\prime}$. Clearly there exists a positive integer $t$ such that $H \subseteq N_{1} \oplus \cdots \oplus N_{t}$. Let $x \in L^{\prime}$. Then $x=x_{1}+\cdots+x_{n}$ for some $n \geq 1$ and $x_{i} \in N_{i}(1 \leq i \leq n)$. Note that $x\left(P_{1} \cap \cdots \cap P_{t}\right) \subseteq N_{t+1} \oplus \cdots \oplus N_{n}$, and hence $x\left(P_{1} \cap \cdots \cap P_{t}\right) \cap H=0$. Thus $x\left(P_{1} \cap \cdots \cap P_{t}\right)=0$. It follows that $L^{\prime}\left(P_{1} \cap \cdots \cap P_{t}\right)=0$.

Let $j \geq t+1$. Let $x \in N_{j}, x \notin K_{j}$. Now $x=y-y^{\prime}$ for some $y \in L, y^{\prime} \in L^{\prime}$. Note that $y \in K_{1} \oplus K_{2} \oplus K_{3} \oplus \cdots$, so that $y=k_{1}+\cdots+k_{s}$ for some $s \geq 1$ and 
$k_{i} \in K_{i}(1 \leq i \leq s)$. Note that $y^{\prime} \in L^{\prime}$, so that $y^{\prime}\left(P_{1} \cap \cdots \cap P_{t}\right)=0$, and hence $\left(k_{j}-x\right)\left(P_{1} \cap \cdots \cap P_{t}\right)=0$. It follows that $P_{1} \cap \cdots \cap P_{t} \subseteq P_{j}$, because $k_{j}-x \neq 0$. Thus $Q=P_{1} \cap \cdots \cap P_{t} \subseteq P_{j}$ for all $j \geq 1$.

Let $N=N_{1} \oplus N_{2} \oplus N_{3} \oplus \cdots$. Note that $N Q=0$ and $Q$ is an essential right ideal of $R$. In particular, $Q \neq 0$. By Lemma 2.2(iii) the $(R / Q)$-module $N$ is a $d^{*} \mathscr{U}$-module with zero socle, and by assumption, $N \in \mathscr{U}$, a contradiction. This completes the proof.

\section{References}

[1] I. A]-Khazzi and P. F. Smith, 'Modules with chain conditions on superfluous submodules', Comm. Algebra 19 (1991), 2331-2351.

[2] F. W. Anderson and K. R. Fuller, Rings and categories of modules (Springer, Berlin, 1974).

[3] A. W. Chatters, 'A characterization of right Noetherian rings', Quart. J. Math. Oxford Ser. (2) 33 (1982), 65-69.

[4] R. Gordon and J. C. Robson, 'Krull dimension', Mem. Amer. Math. Soc. 133 (Amer. Math. Soc., Providence, 1973).

[5] D. van Huynh and P. Dan, 'On rings with restricted minimum condition', Arch. Math. (Basel) 51 (1988), 313-326.

[6] J. C. McConnell and J. C. Robson, Noncommutative Noetherian rings (Wiley, New York, 1987).

[7] P. F. Smith, 'Some rings which are characterised by their finitely generated modules', Quart. J. Math. Oxford Ser. (2) 29 (1978), 101-109.

[8] P. F. Smith, 'Modules with many direct summands', Osaka J. Math. 27 (1990), 253-264.

[9] P. F. Smith, D. van Huynh and N. V. Dung, 'A characterization of Noetherian modules', Quart. J. Math. Oxford Ser. (2) 41 (1990), 225-235.

\section{Department of Mathematics}

University of Glasgow

Glasgow G12 8QW

Scotland, UK 